\title{
Our inherent need for associative coherence
}

\author{
Yael Afiki and Moshe Bar
}

7 February 2020

The Gonda Multidisciplinary Brain Research Center, Bar-Ilan University, Ramat Gan, 52900, Israel

Author Note

Correspondence concerning this article should be addressed to Yael Afiki, The Leslie and Susan Gonda Multidisciplinary Brain Research Center, Bar-Ilan University, RamatGann 52900, Israel. E-mail: Yael.Afiki@gmail.com 
Our inherent need for associative coherence

\begin{abstract}
The world around us consists of typical settings manifested as statistical regularities and stored as associations. These associations are beneficial for performance and serve as a source of stability in our perception of a coherent surrounding. What happens when such associativity is not apparent? We presented pairs of associated images and pairs of nonassociated images and compared their corresponding effect on subsequent performance in three different visual perception paradigms: contrast-sensitivity, global vs. local perception, and critical-flicker-fusion. In all three experiments, the performance was significantly inferior when preceded by images with no clear associative connection. We argue that these results reflect our inherent need to find coherence in our environment. When it is not easily detected, we continue to seek an associative link, which in our case persisted and posed a cognitive load on subsequent performance.
\end{abstract}

Keywords: visual performance, associative thinking, mood, cognitive load, contrast sensitivity, global and local perception, critical flicker fusion. 
Our inherent need for associative coherence

The associations of what happens with what, what happens after what, and other regularities found in everyday contexts provide the platform for our stable world model. Those learned associations help us predict what to expect, how to plan, what to approach and what to avoid (Bar, 2004). We therefore constantly work to find, store and utilize associations on an ongoing, though not necessarily conscious, basis. The associative nature of our world is also reflected in the associative nature of our thought.

A thinking pattern that is associative advances from one concept to an associated concept regularly, coherently, and seamlessly can significantly affect cognitive performance. For example, there is a strong link between associative abilities and creativity (Levin, 1978; Mednick, Mednick, \& Jung, 1964), and associative thinking contributes to problem-solving and critical thinking skills (Rowe, 1991). Additionally, associative information can serve as a platform for generating predictions (Bar, 2007), which may be why associations are found to be intrinsically attractive (Trapp, Shenhav, Bitzer, \& Bar, 2015).

Associative thinking also affects various aspects of attention and memory. Specifically, associated objects may attract more attention (Chun, 2000), which can provide an easier path to perceptual awareness and working memory (Sperling, 1960). Indeed, during visual search performance tasks, for example, associations between objects influence eye movements, recall, recognition, and the accuracy of the report (Moores, Laiti, \& Chelazzi, 2003).

The effect of associations, especially contextual, on recognition of scenes and objects has also been examined extensively (Bar, 2004; Biederman, 1972). Thus, the facilitatory involvement of associations in memory encoding, retrieval, and in the generation of predictions is already widely acknowledged. 
Our inherent need for associative coherence

Given how much we rely on associations, it is reasonable to suspect that we actively look for them in our environment. The question is to what extent, and at what possible costs. We presented pairs of associated images (e.g., monkey-banana) and pairs of non-associated images (e.g., grapes-flag), and compared their respective influence on subsequent performance in three experiments using three different visual paradigms: 1 . Contrast sensitivity, 2. Global vs. local perception and 3. Critical flicker fusion. We focus on the effect of the presence of a coherent everyday association, or its lack thereof, on perception. The search for an associative link could extend for longer that previously appreciated, even when such a link is not readily apparent.

If visual perception is superior following associative pairs compared with nonassociative pairs, such difference could stem from one of two sources: either associated pairs increase global levels of attention, following the studies reviewed above, thereby improving subsequent performance, or the non-associated pairs trigger a sustained attempt to nevertheless seek an associative link, thereby diminishing the resources available for the subsequent task. These two conditions were compared with a control condition that was meant to help distinguish the source of any difference in subsequent performance

\section{Shared Materials and Methods}

We used a list of word associations (Rubinsten, Anaki, Henik, Drori, \& Faran, 2005). The list of associative strength was created by presenting participants with a target word and asking them to name the first word that comes to their mind. The response consensus of participants who gave the second word of the pair as the first response to the first word across participants was used to compute associative strength. We used only word pairs that represented concrete nouns that could be translated into images (e.g., cow 
Our inherent need for associative coherence

and pen). We translated each word to picture and presented image pairs instead of word pairs.

For non-associated image pairs, using the associated word pairs list, we presented two images that did not appear as an oppositional option of any individual. All the stimuli were taken from Internet sources. The images were modified in Photoshop CS6. The stimuli were cut from their original background and inserted into a white (RGB: 255, 255, 255) background.

To make sure that image pairs have an associative relation as words (or nonrelation), a separate group of participants (twenty Bar-Ilan University students, 15 females, age range $22-29$, mean age $=23.84$ ) were asked to rate, in their opinion, to what extent the images are related to each other. The rating range was a number between 0 and 10 (highest). Image pairs that received an average of 8.5 points and above were set as associated image pairs (the mean score was 8.9). In contrast, image pairs that received an average of 1.5 points and below were assigned as non-associated image pairs (the mean score was 1.2). Image pairs with a rating in between were discarded from the experiment. We used the same pairs in all three experiments. The Bar-Ilan Brain Research Ethics Committee approved all the studies reported. All participants provided informed written consent before starting the experiments. Different participants participated in the different experiments.

\section{Experiment 1 - Associations and contrast sensitivity}

Contrast sensitivity (CS) measures the ability to detect differences between light and dark bands with no clear boundary (Kelly, 1977). To test the hypothesis that associative coherence might affect perception, in this experiment we measured how associative processing may affect detection thresholds in a staircase CS task by comparing 
Our inherent need for associative coherence

performance when CS task was preceded by associative pairs, non-associative pairs, and baseline control where no images preceded the CS task.

\section{Participants}

Forty-one Bar-Ilan University students with normal or corrected-to-normal visual acuity and with no ocular pathology $(24$ females, age range $19-32$, mean age $=24.17)$ participated in the experiment for credit toward a course requirement [In all the experiments reported here, our target sample size was chosen to be above the sample sizes in other recent studies involving associative processing (Bar-Anan, Liberman, \& Trope, 2006; Moores et al., 2003; Trapp et al., 2015)]. One participant was disqualified based on his incorrect responses during catch trials.

\section{Stimuli}

Three hundred and eight pictures of neutral objects were used in 147 image pairs and 14 individual images. The target display was a Gabor patch, 2 cycles-per-degree (cpd). The Gabor was slanted $\pm 45^{\circ}$ from the vertical.

\section{Procedures}

In all three experiments, stimuli were presented on a PC with a resolution of $1920 X 1080$ pixels and were controlled by a custom program written in MatLab (MathWorks, Natick, MA) using Psychtoolbox (Brainard \& Vision, 1997) and were run on a Dell computer. The head of each participant was stabilized with an adjustable padded fixture, $55 \mathrm{~cm}$ from the computer screen.

The experiment began with a practice phase that consisted of seven image pairs followed by a Gabor patch. The experiment itself was divided into three blocks (baseline, associated, and non-associated block). Each block consisted of 70 trials. The associated 
Our inherent need for associative coherence

and non-associated blocks consisted also 7 catch trials which were used to verify the performance of the task (a total of 77 trials each; 70 experimental trials, 7 catch trials). The baseline block did not consist of image pairs, but rather only the Gabor judgments. Participants participated in all three blocks, one after the other. Each pair was seen only once during the experiment.

Before the experiment began, participants were given instructions about the general nature of the task and were instructed to attend to the objects. No instructions were given about the nature of the images or the possible relations between them.

During the baseline block, using an adaptive staircase method (explained below), a Gabor pattern was presented in the center of the screen for $150 \mathrm{~ms}$ ( $54 \mathrm{X} 54$ pixels). The participants required to determine the orientation of the Gabor pattern and respond as quickly as possible by pressing the "Right Arrow" if they thought the Gabor was slanted right, or by pressing the "Left Arrow" for a slanted left. The purpose of the task is for contrast changes.

During the associated and non-associated blocks, two images were presented consecutively in the center of the screen (54X54 pixels). A Gabor pattern, using an adaptive staircase method, was then presented also in the center of the screen (54X54 pixels, $2 \mathrm{cpd}$ ). Each trial consisted of seven presentations (Fig. 1): a fixation cross (500 $\mathrm{ms}$ ), an object image (300 ms), another fixation cross (250 ms), a second object image (300 ms), a fixation cross (250 ms), a Gabor image (150 ms), and a question mark for a response. The pairs of objects could be either associated (e.g., bow-arrow) or nonassociated (e.g., flower-phone) according to the characteristics of the block. The Gabor was slanted $\pm 45^{\circ}$ from the vertical. The participants required to determine the orientation of the Gabor pattern, and respond as quickly as possible by pressing the "Right Arrow" if 
Our inherent need for associative coherence

they thought the Gabor was slanted right, or by pressing the "Left Arrow" for a slanted left.

To ensure that participants attend the object pairs, we added seven catch trials in each block. In catch trials, two identical images presented consecutively in the center of the screen. After the Gabor pattern was presented, participants required to ignore the orientation of the Gabor and press the "Down Arrow". This task presumably increased attention equally in both blocks.

All stimuli were presented randomly. The order of the blocks (baseline, associated, and non-associated) was randomized across participants. The time elapsed from stimulus onset to response was computed on-line for each trial and was stored as reaction time $(\mathrm{RT})$ for that contrast. If the response was incorrect, the participant's response was disqualified from the RT analysis. If a participant had more than $50 \%$ errors in the catch trials, the participant was omitted from the analysis. 


\section{Single trial}

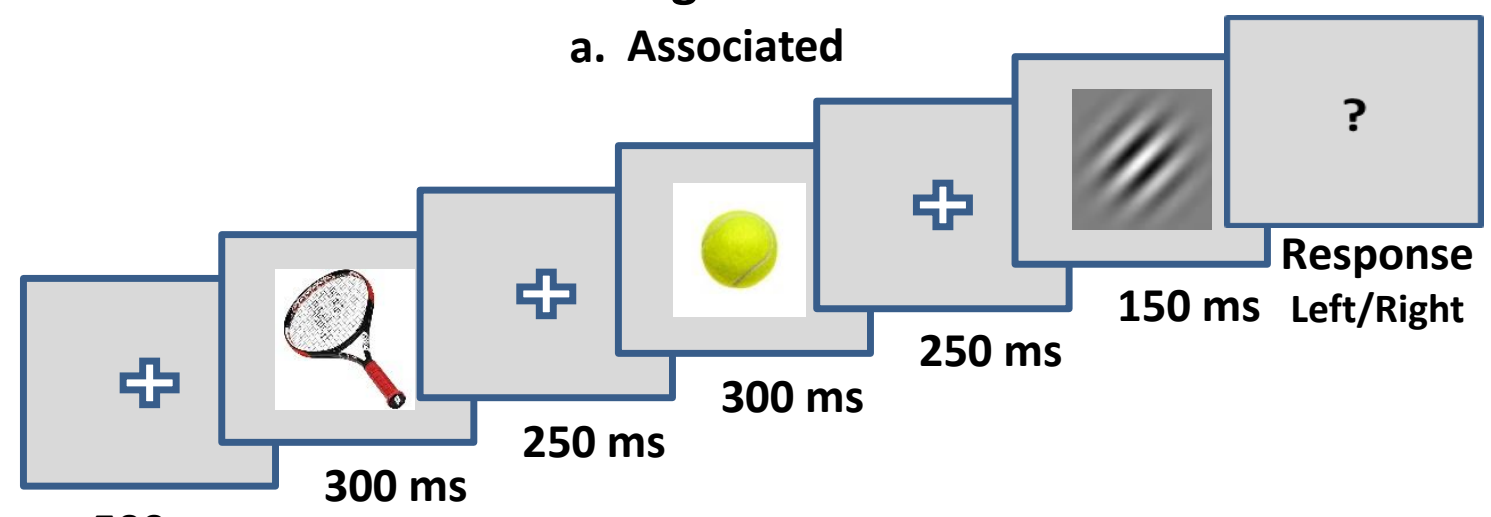

\section{$500 \mathrm{~ms}$}

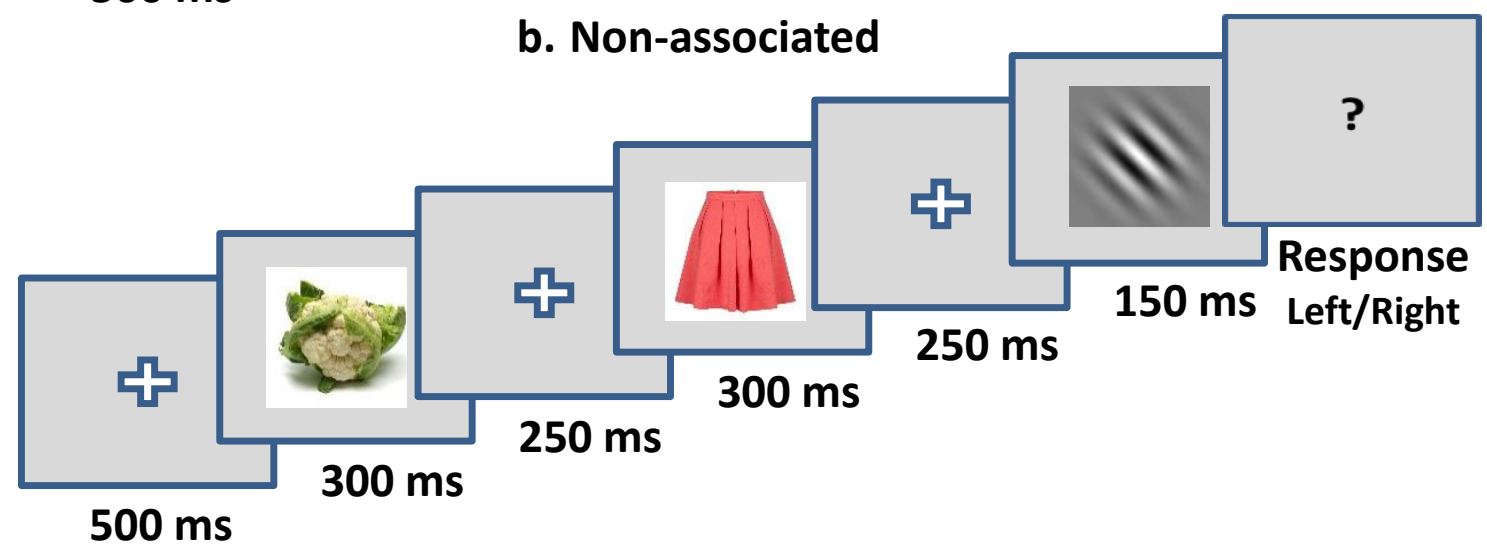

Figure 1. Illustration of the paradigm. The experiment was divided into three blocks. Here we illustrate the Associated block (a) and the Non-associated block (b). Each trial consisted of seven presentations. Participants were required to attend the objects, and then to determine the orientation of the subsequent Gabor pattern.

An adaptive psychophysical staircase procedure (QUEST) was used in all three blocks to estimate individual sensitivity thresholds. The staircase procedure was identical to the experimental procedure described at Watson and Pelli (1983). Thresholds were measured using the Quest toolbox from MatLab. The performance level of the estimated threshold was set at $75 \%$ of detected stimuli. In QUEST, the algorithm determines the probability density function (pdf) of a correct discrimination response of the participant as well as the next stimulus intensity parameter to be presented, based on the response to previous trials (Farell \& Pelli, 1999; Watson \& Pelli, 1983). The estimated parameters of this distribution are updated after each trial according to the participant's performance, and a new intensity parameter is set up for the next trial. If the participant had recognized 
Our inherent need for associative coherence

the stimulus, the new intensity parameter moved one step down (i.e., made harder). If, however, the participant had failed to recognize the stimulus, the new intensity parameter moved one step up (i.e., made easier). At the end of the trials, Quest provided a final log threshold estimate, which was the mean of the pdf in intensity scale (corresponds to $\log _{10}$ contrast), and it set as the CS threshold of the participant (Farell \& Pelli, 1999; Watson \& Pelli, 1983). Feedback was given for both correct and for incorrect responses.

\section{Results}

The data from the experiment were analyzed to determine whether there were differences in the mean CS threshold between the blocks (baseline, associated, and nonassociated). Behavioral statistical analyses for all experiments were carried out using Statistical Package for the Social Sciences (SPSS). The Bayes Factor algorithm was implemented with JASP statistical software using Cauchy a prior of 0.707 .

To test for mean differences in scores between blocks, we conducted a repeated measurements ANOVA with baseline, associated and non-associated blocks as the independent variable. This analysis revealed a main effect in the mean CS threshold between conditions (baseline block $=0.00903$ log intensity; associated block $=0.01090$ $\log$ intensity; non-associated block $=0.01494 \log$ intensity; $F(2,78)=23.019, p<$ $0.00001, \eta^{2}=0.371$, observed power $\left.=1\right)$. The mean CS threshold were plotted as a function of the blocks (Fig. 2a). Pairwise comparisons analysis (using Bonferroni correction) revealed significant difference between the baseline and the non-associated block ( $p<0.00001$, Cohen's $d=1.013,95 \%$ Confidence Interval for mean Difference (CI) $[0.004,0.008])$. Also, a significant difference between the associated and the nonassociated block was found ( $p=0.000044$, Cohen's $d=0.782$, CI $[0.002,0.006])$. However, there was no significant difference between the baseline and the associated 
Our inherent need for associative coherence

block ( $p=0.153$, Cohen's $d=0.318$, CI [-0.0042, 0.00045]), and therefore our subsequent analyses included only the associated and the non-associated blocks. These showed that the mean CS threshold in the non-associated block was higher (implying poorer visual performance; results in $\log$ intensity units) than in the associated block (Fig. 2a). Additionally, we conducted a paired t-test for analysis of reaction time between the associated and the non-associated block. The analysis revealed that the reaction time was significantly longer in the non-associated block than for the associated block (associated block $=850.725 \mathrm{~ms} ;$ non-associated block $=868.192 \mathrm{~ms} ;$ two-tailed $t(39)=2.134, p=$ 0.0391, Cohen's $d=0.337$, CI [0.911, 34.023], Bayes Factor $\left(B F_{10}\right)=1.302$, Fig. $\left.2 b\right)$.

We further tested whether the order of the blocks affected the results, with the intention to see whether the first block induced a state of mind (e.g., associative) that was then carried to the subsequent blocks. To do that, we calculated the differences between the thresholds of each block for each participant and contrasted the results based on whether the starting block was the associated or the non-associated block. A paired t-test revealed no significant difference in the difference of threshold between the blocks (starting block: associated block $=0.0028$ log intensity; non-associated block $=0.0052$ $\log$ intensity; two-tailed $t(19)=1.95, p=0.066$, Cohen's $d=0.436$, CI [-0.0051, 0.00018], $B F_{10}=1.119$, Fig. $2 \mathrm{c}$ ), meaning, the starting block did not affect the results. 


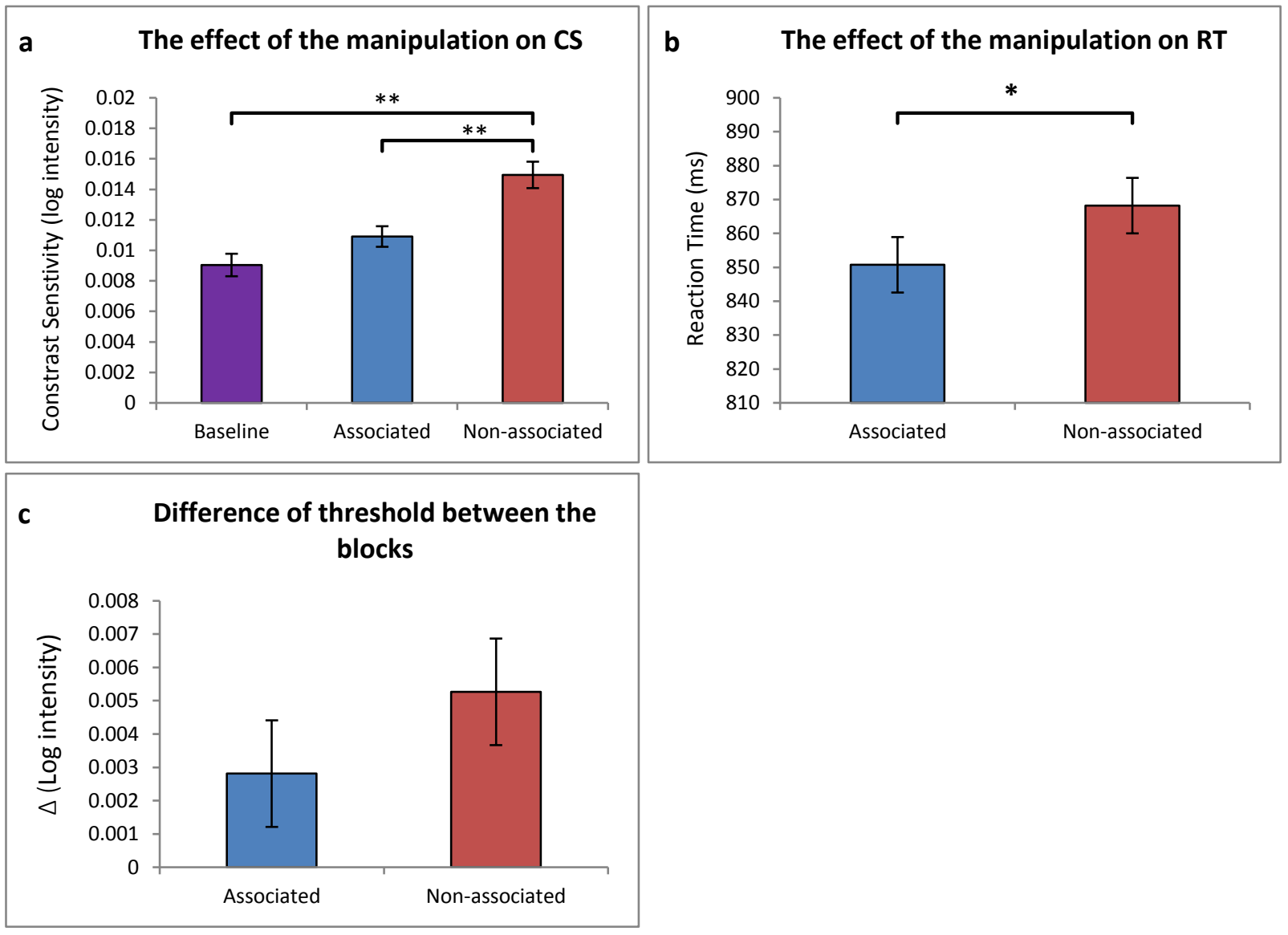

Figure 2. Contrast sensitivity results. a. The results of the three blocks (baseline, associated, and non-associated). Results presented in log intensity units. CS threshold was found to be lower in the associated block, indicates better visual performance. No significant difference was found between the baseline and the associated block. $b$. The reaction time was found to be shorter in the associated block. c. No significant difference was found in the difference of threshold between the blocks indicates that the starting block did not affect the results. Purple, Baseline; Blue, Associated block; Red, Nonassociated block. Error bars indicate standard error of the mean, and asterisks indicate the significant differences between conditions $(* * p<.001, * p<.05)$.

CS defines the threshold for detection of the minimum perceptible contrast, and here we tested whether this threshold is influenced by associative coherence, or its absence. The results support this notion in that the lack of associative coherence reduced contrast sensitivity while associative processing did not affect contrast sensitivity beyond that of baseline. These results favor the explanation that the lack of associative coherence elicited a lasting attempt to still find a link, which continued into and hampered the Gabor judgment, rather than the explanation that associated pairs increased attentional allocation because then the associated blocks would have shown sensitivity that is higher than 
Our inherent need for associative coherence

baseline. In the next two experiments, we tested the same hypothesis for two other representative aspects of visual perception.

\section{Experiment 2 - Associations and hierarchical perception}

The development of object recognition is widely believed to be a hierarchical process, progressing from global to local properties (Kimchi, 1992; Navon, 1977). Operationally, local processing is based on selective attention to individual elements of an object or a scene, whereas global processing involves integrating spatial-local elements by linking them together into a larger form of a global structure (Kimchi, 1992). That processing of the global form typically precedes the local form (Navon, 1977) was dubbed the global precedence effect. Similarly, it is argued that the global properties, conveyed mainly by low spatial frequencies, trigger top-down facilitation predictions in visual recognition (Bar et al., 2006).

In this experiment, we evaluated how associative processing (or its lack thereof) can affect global vs. local perception. It has been shown that cognitive load diminishes the precedence of global information (Hoar \& Linnell, 2013). Therefore, if the search for an associative link even for the non-associated pairs continues into the visual task (Global vs. Local in this case), we would expect to see less of a global precedence effect in the non-associative condition.

\section{Materials and Methods}

\section{Participants}

Forty Bar-Ilan University students with normal or corrected-to-normal visual acuity and with no ocular pathology (27 females, age range $18-32$, mean age $=24.05$ ) participated in the experiment for credit toward a course requirement. 
Our inherent need for associative coherence

\section{Stimuli}

Five hundred ninety-four pictures of neutral objects were used in 276 image pairs and 42 individual images. The target display was a 'Navon' stimulus. The inconsistent Navon set of stimuli consisted of compound letters consisting of several numbers of lower case Ss or Hs (14 lower case Ss, 12 lower case Hs; local letters) configured to form of a global H or S ('Navon' stimuli), respectively. The consistent Navon set of stimuli consisted of a global $\mathrm{H}$ formed by lower case Hs and a global $\mathrm{S}$ formed by lower case Hs. The global letters subtended $3 \times 2$ degrees of visual angle, and the smaller local letters were 0.4x0.35 degrees of visual angle. Each letter was displayed 54 times per block.

\section{Procedures}

The experiment began with a practice phase that consisted of 12 image pairs followed by Navon stimuli. The experiment was divided into three blocks (baseline, associated, and non-associated block). Each block consisted of 216 trials. The associated and non-associated blocks consisted also 21 catch trials which were used to verify the performance of the task (a total of 237 trials each; 216 experimental trials, 21 catch trials). The baseline block did not consist of image pairs, but rather only a Navon Stimulus. Participants participated in all three blocks, one after the other. Each pair was seen no more than twice during the experiment.

Before the experiment began, participants were given instructions about the general nature of the task and were instructed to attend to the objects. No instructions were given about the nature of the images or the possible relations between them.

During the baseline block, a Navon stimulus was presented in the center of the screen for $40 \mathrm{~ms}$. Participants had to judge which letter they perceived first and respond 
Our inherent need for associative coherence

to it as quickly as possible by pressing "S" if the perception was the letter s or by pressing "H" if the perception was the letter h.

During the associated and non-associated blocks, two images were presented consecutively in the center of the screen (54X54 pixels). A Navon stimulus was subsequently also presented in the center of the screen. Each trial consisted of seven presentations (Fig. 3): a fixation cross (500 ms), an object image (300 ms), another fixation cross $(250 \mathrm{~ms})$, a second object image (300 ms), a fixation cross $(250 \mathrm{~ms})$, a Navon stimulus (40 ms), and a question mark for a response. The pairs of objects could be either associated or non-associated according to the characteristics of the block. The Navon stimuli (Navon, 1977) consisting of a global letter formed by the configuration of local letters (e.g., a global S composed of lower case Hs or a global H composed of lower case Hs). Participants had to judge, in their opinion, which letter they perceived first and respond to it as quickly as possible by pressing "S" if the perception was the letter $\mathrm{s}$ (global or local) or by pressing "H" if the perception was the letter h (global or local).

To ensure participants attend to the objects, we added 21 catch trials in each block. In catch trials, two identical images presented consecutively in the center of the screen. After the Navon stimuli were presented, participants were required to ignore it and to press the "Space". All stimuli were presented randomly. The order of the blocks (baseline, associated, and non-associated) was randomized and counterbalanced across participants. No feedback was given because there was no correct or incorrect response in this experiment. The time elapsed from the stimulus onset to response was computed on-line for each trial and was stored as the RT and response. If a participant committed more than $50 \%$ errors in the catch trials, the participant was omitted from the analysis. 

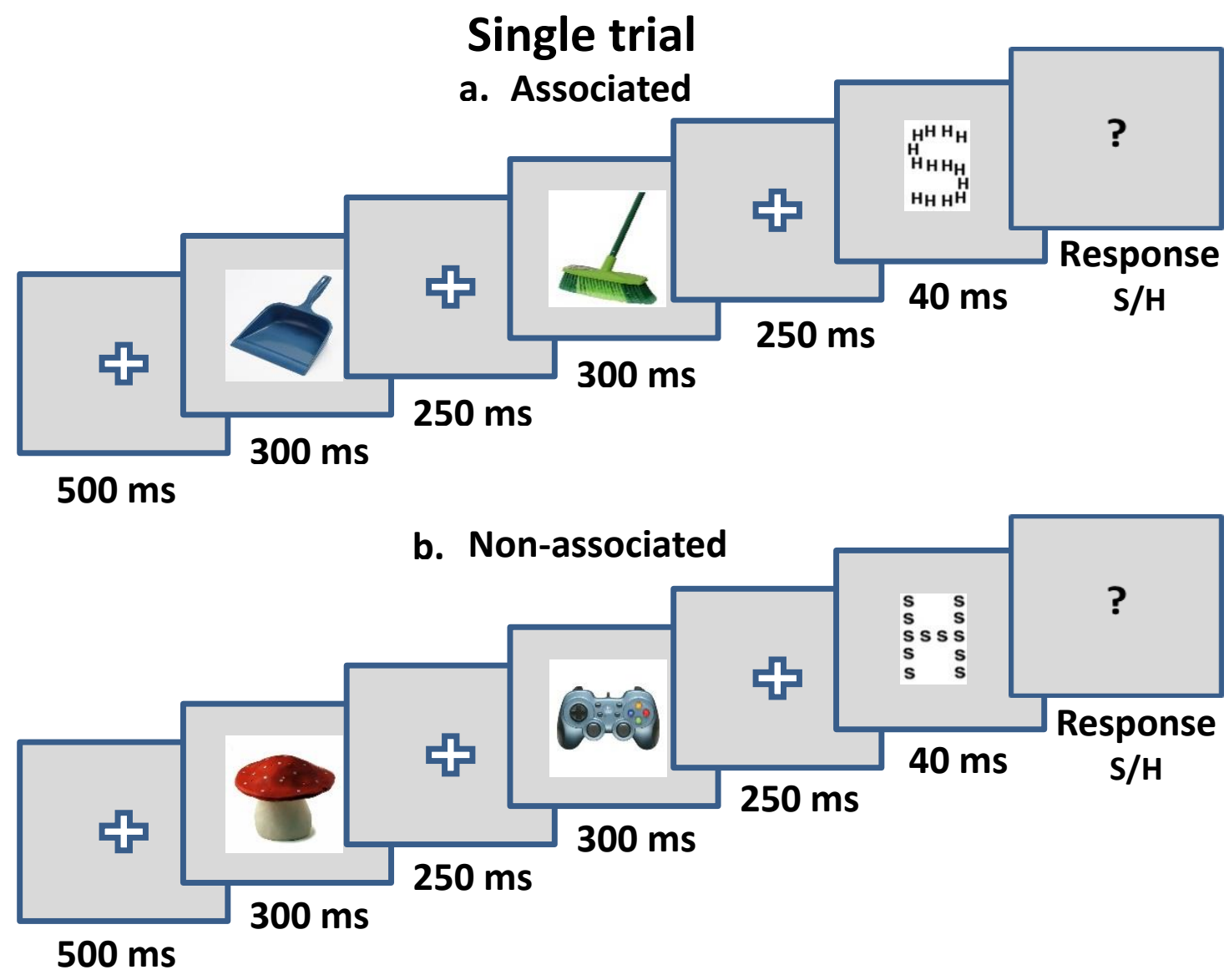

Figure 3. Illustration of the paradigm. The experiment was divided into 3 blocks. Here we illustrated the Associated block (a) and the Non-associated block(b). Each trial consisted of seven presentations. Participants were required to judge, in their opinion, which letter they perceived first.

\section{Results}

The data from the experiment were analyzed to determine whether there were differences in the number of times that the participant chose the global property between the blocks (baseline, associated, and non-associated).

The test score was calculated by the number of times that the participant chose the global property. Processing precedence was calculated by the number of times the participant chose the global property relative to the total number of attempts. To test for mean differences in scores between blocks, we conducted a repeated measurements ANOVA with baseline, associated and non-associated blocks as the independent variable. 
Our inherent need for associative coherence

This analysis revealed a main effect in the number of times that the participant chose the global property between conditions (baseline block $=79.14 \%$; associated block = $80.88 \%$; non-associated blocks $=69.86 \% ; F(2,78)=7.811, p=0.00081, \eta^{2}=0.167$, observed power $=0.944$ ). The mean numbers of times that the participant chose the global property are plotted as a function of the blocks (Fig. 4a). Pairwise comparisons analysis (using Bonferroni correction) revealed a significant difference between the baseline and the non-associated block ( $p=0.048$, Cohen's $d=0.398$, CI $[0.0655,18.499])$. Also, a significant difference between the associated and the non-associated block was found ( $p$ $=0.0039$, Cohen's $d=0.547$, CI $[3.046,18.990])$. However, like in the previous experiment, there was no significant difference between the baseline and the associated block ( $p=1$, Cohen's $d=0.153$, CI $[-6.234,2.762])$, and therefore our subsequent analyses included only the associated and the non-associated blocks. The results showed that the number of times that participants chose the global property in the non-associated block was lower than in the associated block (Fig. 4a). Additionally, we conducted a paired t-test for analysis of reaction time between the associated and the non-associated block. The analysis revealed that the reaction time was significantly longer for a global property in the non-associated block than for the associated block (associated block = $447.13 \mathrm{~ms}$; non-associated block $=500.50 \mathrm{~ms}$; two-tailed $t(39)=2.234, p=0.031$, Cohen's $d=0.535$, CI [5.037, 101.70], $B F_{10}=1.564$, Fig. $\left.4 \mathrm{~b}\right)$.

Like in the previous experiment, and following the same reason, we tested whether the order of the blocks affected the results. We calculated the differences in choosing the global property in each block for each participant and contrasted the results based on whether the starting block was the associated or the non-associated block. A paired t-test revealed no significant difference in choosing the global property between the blocks (starting block: associated block $=12.40 \%$; non-associated block $=9.63 \%$; two- 
tailed $t(19)=0.411, p=0.685$, Cohen's $d=0.092$, CI $[-11.35,16.91], B F_{10}=0.251$, Fig.

4c), meaning, like we showed in the previous experiment, the starting block did not affect the results.
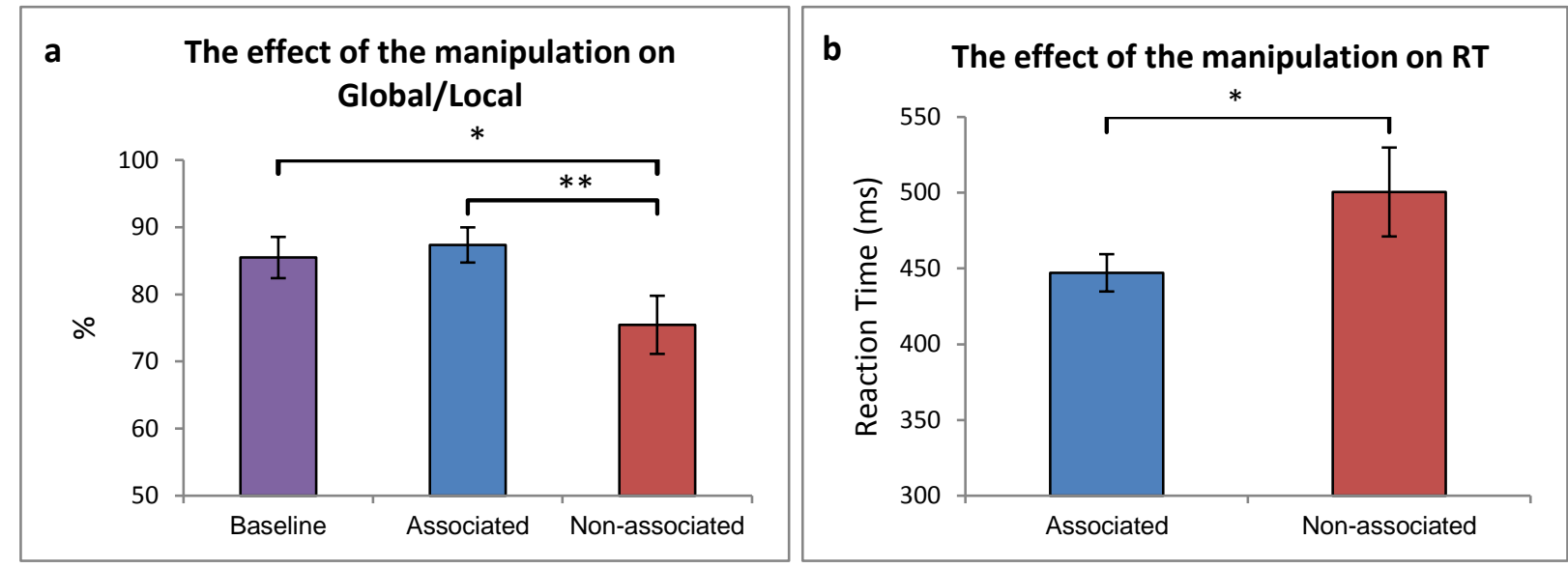

\section{c Difference in choosing the global property between the blocks}

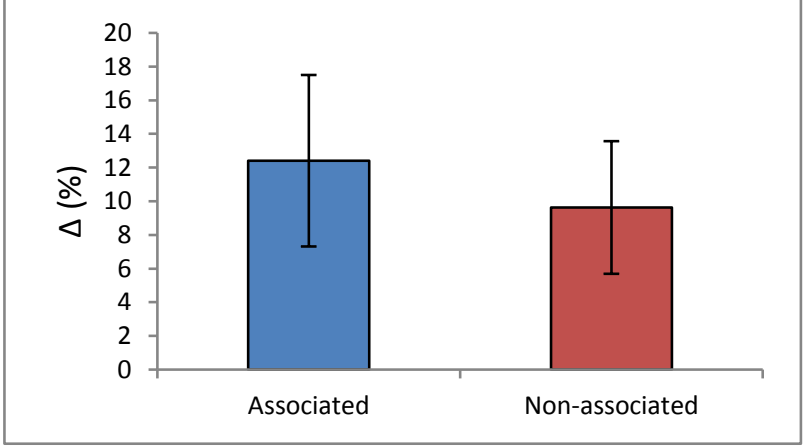

Figure 4. Global vs. local results. a. The results of the 3 blocks (baseline, associated, and non-associated). The number of times that participants chose the global property over the local property was higher in the associated block. No significant difference was found between the baseline and the associated block. The results in \%. b. The reaction time was found shorter in the associated block. c. No significant difference was found in the number of times that participants chose the global property between the blocks indicates that the starting block did not affect the results. Purple, Baseline; Blue, Associated block; Red, Non-associated block. Error bars indicate the standard error of the mean, and asterisks indicate significant differences between conditions $(* * p<.001, * p<.05)$.

These results are similar in vein to what we found in the previous experiment, whereby a lack of an associative link results in a subsequently degraded performance, presumably because of the cognitive load that a continued search for an associative link pose. In the third experiment, we tested for a possible effect of lack of associativity on yet another aspect of low-level visual perception. 
Our inherent need for associative coherence

\section{Experiment 3 - Associations and critical flicker fusion}

The visual system processes visual information in both spatial and temporal domains. In

Experiment 1 we addressed spatial resolution when we examined the influence of association on contrast sensitivity, which is defined as the ability to discriminate, in space, between two adjacent objects. In this experiment, we test temporal resolution through the ability to discern luminance changes over time. Critical Flickering Fusion (CFF), the lowest frequency rate (measured in $\mathrm{Hz}$ ) at which intermittently presented light stimuli appear to be steady and continuous for the average human observer, is widely used for assessing temporal processing in the visual system (Simonson \& Brozek, 1952). When light is flickering at a higher or equal value of the CFF threshold, the flashes cannot be distinguished from a steady projection, through visual persistence (Kalloniatis \& Luu, 2007; Simonson \& Brozek, 1952). Conversely, below the CFF threshold, individual flashes may be discriminated from steady lights (Kalloniatis \& Luu, 2007; Simonson \& Brozek, 1952). In this experiment, we tested how associativity might interact with low-level CFF perception.

\section{Materials and Methods}

\section{Participants}

Forty Bar-Ilan University students with normal or corrected-to-normal visual acuity and with no ocular pathology ( 25 females, age range $18-33$, mean age $=23.40$ ) participated in the experiment for credit toward a course requirement.

\section{Stimuli}


Our inherent need for associative coherence

Five hundred and eighteen pictures of neutral objects were used in 247 image pairs and 24 individual images. The target display was a flashing white light-emitting diode (LED).

\section{Procedures}

In the flicker experiment, the participant observes a flashing light. Exposure to glare can cause discomfort to the participant. Also, multiple exposures may cause insensitivity to participants' reactions. Thus, to obtain a reliable response and to accommodate the participants, we decided to minimize the duration of the experiment as much as possible. Therefore, because of the lack of significant differences between the baseline block and the associated block in the two previous experiments, we decided in this experiment to focus only on the associated and the non-associated blocks and not to include a baseline block.

The experiment began with a practice phase that consisted of 7 image pairs followed by a flashed light. The experiments were divided into two blocks (associated and non-associated) of 132 trials each (60 experimental trials, 60 fillers, and 12 catch trials), and the participants participated in both blocks, one after the other. Each pair was seen only once during the experiment.

Before the experiment began, participants were given instructions about the general nature of the task and were instructed to attend to the objects. No instructions were given about the nature of the images or the possible relations between them.

In this experiment, two images were presented in the center of the screen (54X54 pixels) separately. Using an adaptive staircase method (explained below), a LED (5 mm, current $20 \mathrm{~mA}$ ) was then flashed in the center of the screen. An external Arduino 
Our inherent need for associative coherence

microcontroller controlled the flashed light. Each trial consisted of seven presentations (Fig. 5): a fixation cross (500 ms), an object image (300 ms), another fixation cross (250 $\mathrm{ms}$ ), a second object image (300 ms), a fixation cross (250 ms), a flickering light stimulus (100 ms), and a question mark for a response. The pairs of objects could be either associated or non-associated according to the characteristics of the block. The participants had to determine whether the flickering light was continuous or flashing and respond as quickly as possible by pressing "yes" if the light was continuous or by pressing "no" if it was flashing.

To ensure that participants attend the objects, we added 12 catch trials in each block. In catch trials, two identical images presented consecutively in the center of the screen. After the flickering light was presented, participants were required to ignore it and to press the "Space". All stimuli were presented randomly. The order of the associated and non-associated blocks was randomized across participants. The time that elapsed from the stimulus onset to response was computed on-line for each trial and was stored as RT and flashing rate. If a participant had more than $50 \%$ errors in the catch trials, the participant was omitted from the analysis. 

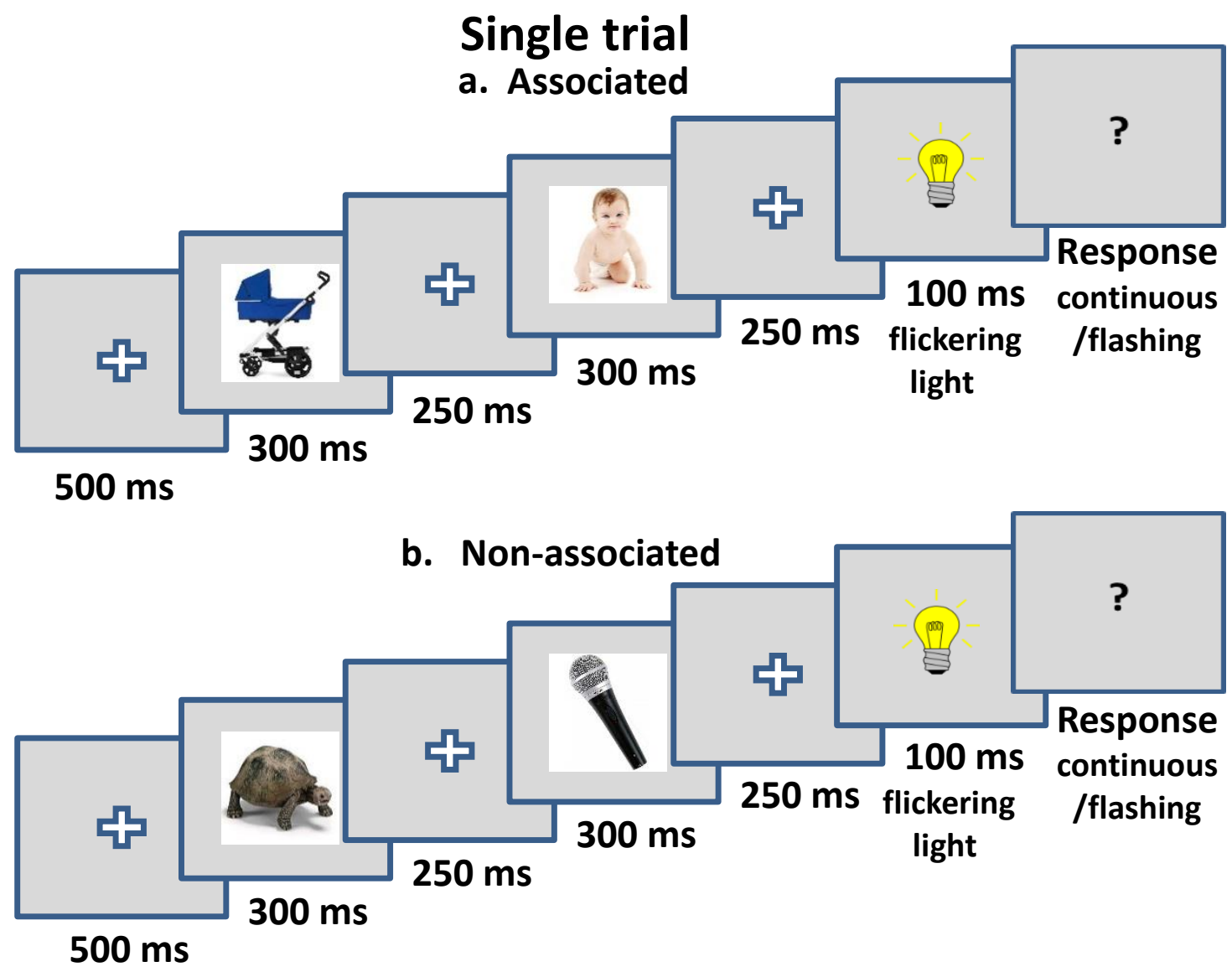

Figure 5. Illustration of the paradigm. The experiment was divided into 2 blocks. a. Associated block. b. Nonassociated block. Each trial consisted of seven presentations. Participants required to determine whether the flickering light is continuous or flashing.

Functional Adaptive Sequential Testing (FAST) was used in both blocks to estimate the measurement of the CFF. FAST is a Matlab toolbox for efficiently estimating thresholds in behavioral experiments. A single threshold estimated to be between 0 to 60 Hz-units, with a detection task using a Logistic function (default) with slope estimated to be between 0.1 and 1 units. FAST method estimated the full psychophysical function utilizing all data points, and, for each trial, selects the stimulus variable that maximizes the certainty about the function parameter values based on fit parameters (threshold and slope) from all preceding trials (Vul, Bergsma, \& MacLeod, 2010). When the participant responded that the light was continuous, the subsequent frequency decreased, and vice versa. This process is repeated until the end of the experiment. At the end of the trials, 
Our inherent need for associative coherence

FAST provided a final CFF threshold. In each block, 60 trials were used to estimate the final CFF threshold. However, because this function is gradual, the participant is expected to press the same response for several consecutive steps. Therefore, to blur the function, each block included 60 additional trials (fillers) in which the flickering light that displayed was random. The order of all trials was randomized (experimental, fillers and catch trials).

No feedback was given because there were no correct or incorrect responses in this experiment.

\section{Results}

The data from the experiment were analyzed to determine whether there were differences in the mean CFF threshold between the blocks (associated vs. nonassociated). Analyses were conducted only on the experimental trials.

To test the mean differences in scores between blocks, we conducted a paired ttest between the associated and the non-associated blocks. The analysis revealed a significant difference in the mean CFF threshold between the blocks. The mean CFF threshold are plotted as a function of the blocks. The mean CFF threshold in the nonassociated block was lower than in the associated block (associated block $=50.04 \mathrm{~Hz}$; non-associated block $=47.65 \mathrm{~Hz}$; two-tailed $t(39)=-2.390, p=0.0217$, Cohen's $d=$ 0.378 , CI $[0.369,4.428], B F_{10}=2.114$, Fig. $\left.6 a\right)$. That means, for example, that after nonassociated pairs, participants tend to see $49 \mathrm{~Hz}$ as a continuous light, whereas after associated pairs, $49 \mathrm{~Hz}$ were perceived as flickering. We found no significant difference between the conditions regarding the reaction time (associated block $=343.85 \mathrm{~ms}$; nonassociated block $=356.90 \mathrm{~ms}$; two-tailed $t(39)=0.746, p=0.459$, Cohen's $d=0.118, \mathrm{CI}$ $[-22.32,48.43], B F_{10}=0.221$, Fig. $\left.6 b\right)$. We can explain these results by the reason that the flickering light was activated continuously for $100 \mathrm{~ms}$. Participants could not reply 
before the flickering light was turned off, and therefore, it is possible that they already formulated a response and only waited to press it.

Like in the previous experiments, we tested whether the order of the blocks would affect the results. We calculated the differences in the mean CFF threshold in each block for each participant and contrasted the results based on whether the starting block was the associated or the non-associated block. A paired t-test revealed no significant difference in the difference of the mean CFF threshold between the blocks (starting block: associated block $=0.91 \mathrm{~Hz} ;$ non-associated block $=3.87 \mathrm{~Hz} ;$ two-tailed $t(19)=1.465, p=0.159$, Cohen's $d=0.327$, CI [-1.269, 7.184], $B F_{10}=0.584$, Fig. 6c), meaning, like we showed in the previous experiment, the starting block did not affect the results.
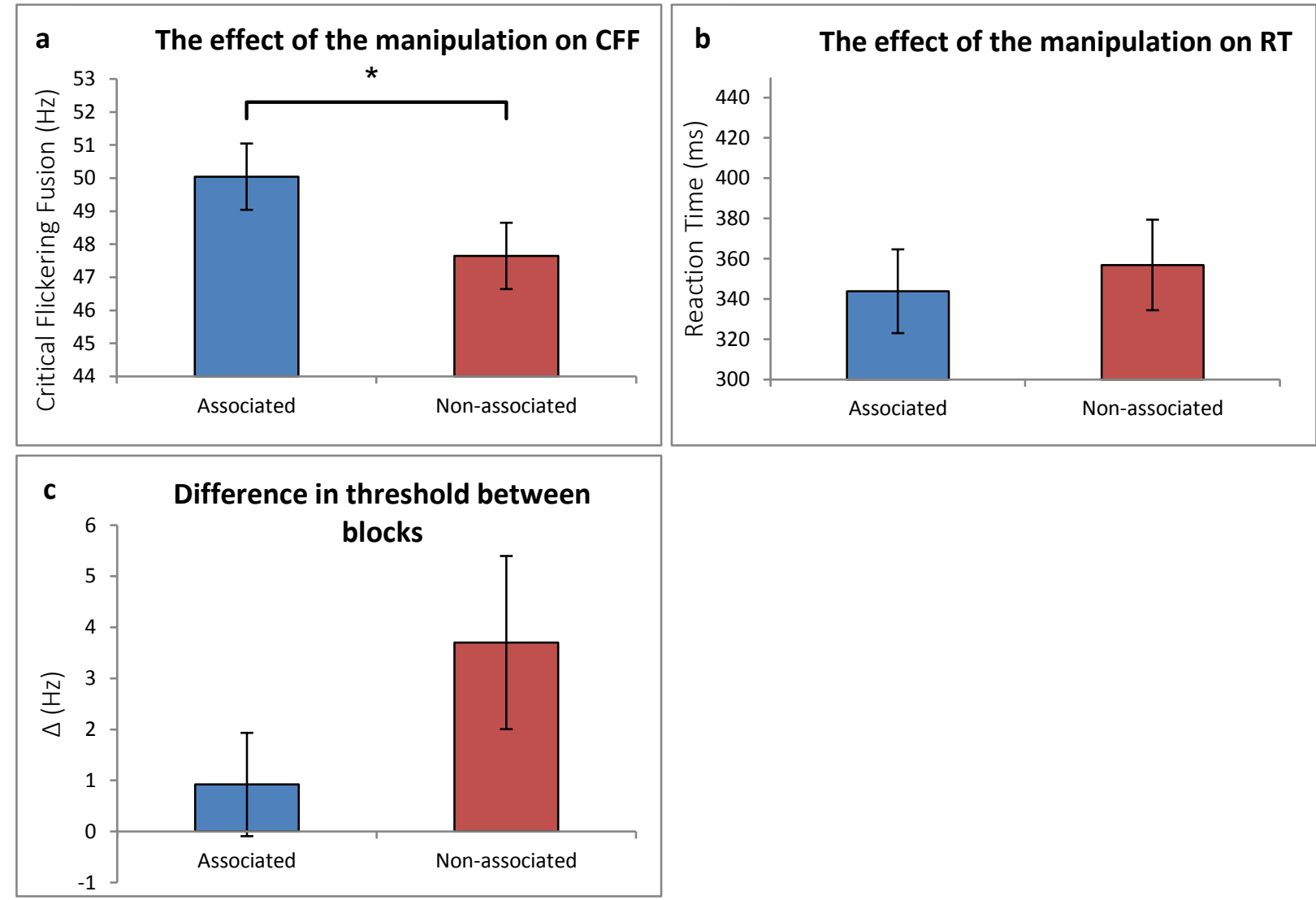
Figure 6. Critical flicker fusion results. a. The mean CFF threshold in the associated block was higher than in the nonassociated block indicates better visual performance. The results in $\mathrm{Hz}$ units. b. No differences were found in reaction times between conditions. c. No significant difference was found in the difference of threshold between the blocks indicate that the starting block did not affect the results. Blue, Associated block; Red, Non-associated block. Error bars indicate the standard error of the mean, and asterisks indicate significant differences between conditions $(* \mathrm{p}<.05)$.

CFF defines the temporal threshold at which a flashing light is perceived as continuous light. The results show that following pairs of images that were not associated with each other the CFF threshold was poorer than following associative pairs, in-line with the outcome of the other two experiments reported here. In other words, seeing two non-associated objects directly reduced subsequent sensitivity in detecting flicker, just like in detecting contrast. Our overarching proposal is that this effect is a result of the cognitive load that a sustained, inherent search for associative relations confers.

\section{Discussion}

In this study, we examined the effects of associative coherence on lower-level perception. We found that a lack of a clear associative coherence significantly degrades subsequent perceptual performance relative to associative processing in the three visual aspects that were tested.

The first experiment revealed a significant increase in CS thresholds (i.e., decreased sensitivity) when it was tested following pairs of non-associated images, compared with pairs of associated images. The second experiment found decreased global precedence for pairs of non-associated images as compared to associated images. The third experiment showed a reduced CFF threshold (i.e., decreased in performance) for a pair of non-associated images. Moreover, in all three visual conditions, RTs were longer following the non-associated pairs (significant only in the first two experiments). In summary, rather than facilitation by association, we observed impairment by a lack of associations. 
Our inherent need for associative coherence

To explain our findings, consider first the spreading activation theory for the organization of memory. According to this theory, concepts are represented in memory as nodes that are interconnected by associative links (Anderson, 1983; Collins \& Loftus, 1975). When we experience an event, nodes that contain information about the event will lead to spreading activation across related nodes and concepts in memory networks. This spreading activation is proposed to occur automatically, quickly, and unconsciously (Neely, 1977; Posner \& Snyder, 1975). According to this account, during our experiments, the first image triggered the activation of representations that are related to this particular image and can follow it next. In the associated block, the image pairs were strongly connected; therefore, we can assume that the second image that was presented was one that was activated by the first image already. This associative match provided the associative coherence, which helped participants move on to the judgment without dwelling on trying to find a connection.

In the non-associated block, on the other hand, the image pairs were unrelated; the first image activated related representations, but the second image did not match any option anticipated based on the spreading activation triggered by the first image. We propose that in this case, when there was no apparent association between the first and the second image of the pair, participants continued to search for one. This persistent search for a link, which is indicative of our inherent need for coherence, has leaked into the subsequent visual task, resulting in diminished resources available for the task at hand. In other words, when there is a clear associative resolution between the images, participants approach the visual task with less of a load and thus more resources for better performance, whereas in the non-associated condition the participants continued to ruminate, or incubate, even if not consciously, on trying to find a link between two unrelated images. 
Our inherent need for associative coherence

The cognitive load theory relates to the amount of information that working memory can hold at one time (Kanfer \& Ackerman, 1989). Since working memory has a limited capacity, if the difference between the total cognitive load and the processing capacity of the working memory is small, then the participant experiences a high cognitive load, and a correspondingly poorer performance (Gigerenzer, 2007). In our experiments, during the non-associated block, we propose that at least some of the cognitive capacity was devoted to finding associative coherence between the pairs of images, and this left significantly reduced capacity to be devoted to subsequent performance.

Numerous studies have shown that visual perception is capacity-limited, and therefore perception relies heavily on the availability of resources (Carmel, Saker, Rees, \& Lavie, 2007; Martens \& Kiefer, 2009). For example, participants in a study by Martens and Kiefer (2009) performed either an easy or a difficult task prior to masked priming in a lexical decision task. The results revealed that after a difficult task, no priming effect was found unless there was a long time-interval between the primary and the secondary task. A study by Carmel and colleagues (2007) similarly found that the detection of temporal patterns also depends on the availability of resources. Their study showed that a high cognitive load might reduce the ability to discriminate between single events, which can lead to impairment in the CFF threshold. Thus, the amount of cognitive load determines the accuracy and the sensitivity of the CFF detection. This supports the result of our Experiment 3 revealed. Additionally, it was found (Baror \& Bar, 2016) that in a free-association task under manipulation of a simultaneous mental load, participants in high load conditions (e.g., keeping in mind a long string of digits) rely heavily on strong immediate associations, while participants in low load conditions (e.g., keeping in mind double-digit numbers) exhibited broader and more unique associations. The high 
Our inherent need for associative coherence

cognitive load apparently captures the available resources and prevented participants from being creative and 'open-minded'.

Although our findings are best explained by the account detailed above, other explanations could be considered. For example, a related account of our findings involves varying the allocation of attention as a function of associations. Studies have shown the value of associative thinking in numerous cognitive and perceptual processes. For instance, it was found that associated objects affect the spread of attention and capture attentional resources (Moores et al., 2003; Summerfield, Lepsien, Gitelman, Mesulam, \& Nobre, 2006). It was suggested that the activation of object representations in working memory triggers associated representations (Downing, 2000; Moores et al., 2003). These representations attract more attention by initiating top-down processes or cause rapid and efficient processing, which attracts attention (Downing, 2000; Sperling, 1960). Therefore, associated images may facilitate subsequent perception by a general increase of attention while non-associated images decrease it, which may be less likely given that baseline and associated conditions showed equivalent results. Nevertheless, in the three experiments, while performing the non-associated block, participants might have been less attentive because they had fewer resources available to manage and perform the task, per the account above, and hence performance significantly decreased.

An alternative account is concerning the link between associations, mood, and performance. A positive mood tends to correlate with broad associative thinking, whereas a negative mood correlates with narrow associative thinking (Bar, 2009; Isen, Johnson, Mertz, \& Robinson, 1985). Moreover, negative mood was found to reduce the resources that are available for effortful processing (Ashby \& Isen, 1999; Fredrickson \& Branigan, 2005). It has been suggested that a reciprocal relation exists between associations and 
Our inherent need for associative coherence

mood (Bar, 2009), whereby mood affects associations and associations affect mood. Indeed, it was found that increasing the breadth of association processing can positively influence mood (Brunyé et al., 2013; Mason \& Bar, 2012).

Another finding that needs to be discussed is the comparison between the baseline and the associated block. The first two experiments included a control baseline block, and a comparison between the baseline and the associated block revealed no significant differences. Examining the current literature indicates that the natural tendency of people is to be exploratory and gravitate to novelty (Baror \& Bar, 2016). In other words, when resources are available, the brain has a basic bias to seek unique associations. It was also found that people are inherently creative in the way they solve problems in daily life (Sternberg, 1999). Also, in studies that estimated the rates of depression and life satisfaction by questions related to subjective well-being found that the majority of people, regardless of nationality, avow positive levels of happiness and well-being (Diener \& Diener, 1996). Furthermore, exploration, creativity, and happiness were found to be an integral part of associated thinking (Baror \& Bar, 2016; Mednick et al., 1964). Associations are considered as a spontaneous, natural, and even default process (Bar, 2007; Baror \& Bar, 2016; Nelson, Bajo, \& Casanueva, 1985; Raaijmakers \& Shiffrin, 1981). Therefore, the induction of associative thinking in healthy people, who are already associative by nature, may not change or promote further associative thinking, which may be one reason why we did not find a difference between the blocks.

In daily life, people rely on associations for most cognitive processes, and look for associative coherence, in the world around us. Associative coherence implies that the world behaves as we expect it to behave, which affirms the internal model of our environment. After all, associations are learned through statistical regularities of what 
Our inherent need for associative coherence

tends to be relevant with what in our world. When we do not find it, we seem to continue to think and search for a link, as our three experiments suggest.

\section{Conclusion}

Perception is shaped and formed by the physical properties of the stimulus, but it is also influenced by factors such as state, attention, context, load, mood, thoughts and beliefs (Balcetis \& Dunning, 2006). This study suggested a novel influence on perception, which is not entirely intuitive, that load imposed by the need to seek coherence even in, and perhaps especially in, non-associative information degrades performance at least on three low-level visual perception tasks. Although the underlying mechanism remains to be elucidated, these findings help advance our understanding of non-physical influences on perception and the power of associative processing to shape our experiences of the world around us.

\section{Author Contributions}

Both authors designed the research. YA carried out the experiments and analyzed the data. Both authors wrote and approved the final version of the manuscript before submission.

\section{Funding}

This research was supported by The Israel Science Foundation (ISF) grant No. 673/17, and by the Sagol Family, both to MB.

\section{References}

Anderson, J. R. (1983). A spreading activation theory of memory. Journal of Verbal Learning and Verbal Behavior, 22(3), 261-295.

Ashby, F. G., \& Isen, A. M. (1999). A neuropsychological theory of positive affect and 
Our inherent need for associative coherence

its influence on cognition. Psychological Review, 106(3), 529.

Balcetis, E., \& Dunning, D. (2006). See what you want to see: motivational influences on visual perception. Journal of Personality and Social Psychology, 91(4), 612.

Bar-Anan, Y., Liberman, N., \& Trope, Y. (2006). The association between psychological distance and construal level: Evidence from an implicit association test. Journal of Experimental Psychology: General, 135(4), 609.

Bar, M. (2004). Visual objects in context. Nature Reviews Neuroscience, 5, 617.

Bar, M. (2007). The proactive brain: using analogies and associations to generate predictions. Trends in Cognitive Sciences, 11(7), 280-289.

Bar, M. (2009). A cognitive neuroscience hypothesis of mood and depression. Trends in Cognitive Sciences, 13(11), 456-463.

Bar, M., Kassam, K. S., Ghuman, A. S., Boshyan, J., Schmid, A. M., Dale, A. M., ... Rosen, B. R. (2006). Top-down facilitation of visual recognition. Proceedings of the National Academy of Sciences of the United States of America, 103(2), 449454.

Baror, S., \& Bar, M. (2016). Associative activation and its relation to exploration and exploitation in the brain. Psychological Science, 27(6), 776-789.

Biederman, I. (1972). Perceiving real-world scenes. Science, 177(4043), 77-80.

Brainard, D. H., \& Vision, S. (1997). The psychophysics toolbox. Spatial Vision, 10, 433-436.

Brunyé, T. T., Gagnon, S. A., Paczynski, M., Shenhav, A., Mahoney, C. R., \& Taylor, H. A. (2013). Happiness by association: Breadth of free association influences affective states. Cognition, 127(1), 93-98.

Carmel, D., Saker, P., Rees, G., \& Lavie, N. (2007). Perceptual load modulates conscious flicker perception. Journal of Vision, 7(14), 14.

Chun, M. M. (2000). Contextual cueing of visual attention. Trends in Cognitive Sciences, 4(5), 170-178.

Collins, A. M., \& Loftus, E. F. (1975). A spreading-activation theory of semantic processing. Psychological Review, 82(6), 407.

Diener, E., \& Diener, C. (1996). Most people are happy. Psychological Science, 7(3), 181-185.

Downing, P. E. (2000). Interactions between visual working memory and selective attention. Psychological Science, 11(6), 467-473.

Farell, B., \& Pelli, D. G. (1999). Psychophysical methods, or how to measure a threshold and why. Vision Research: A Practical Guide to Laboratory Methods, 5, 129-136.

Fredrickson, B. L., \& Branigan, C. (2005). Positive emotions broaden the scope of attention and thought-action repertoires. Cognition \& Emotion, 19(3), 313-332. 
Our inherent need for associative coherence

Gigerenzer, G. (2007). Gut feelings: The intelligence of the unconscious. Penguin.

Hoar, S., \& Linnell, K. J. (2013). Cognitive load eliminates the global perceptual bias for unlimited exposure durations. Attention, Perception, \& Psychophysics, 75(2), 210-215.

Isen, A. M., Johnson, M. M., Mertz, E., \& Robinson, G. F. (1985). The influence of positive affect on the unusualness of word associations. Journal of Personality and Social Psychology, 48(6), 1413.

Kalloniatis, M., \& Luu, C. (2007). Temporal resolution. In Webvision: The Organization of the Retina and Visual System [Internet]. University of Utah Health Sciences Center.

Kanfer, R., \& Ackerman, P. L. (1989). Motivation and cognitive abilities: An integrative/aptitude-treatment interaction approach to skill acquisition. Journal of Applied Psychology, 74(4), 657.

Kelly, D. H. (1977). Visual contrast sensitivity. Optica Acta: International Journal of Optics, 24(2), 107-129.

Kimchi, R. (1992). Primacy of wholistic processing and global/local paradigm: a critical review. Psychological Bulletin, 112(1), 24.

Levin, I. (1978). Creativity and two modes of associative fluency: Chains and stars. Journal of Personality, 46(3), 426-437.

Martens, U., \& Kiefer, M. (2009). Specifying attentional top-down influences on subsequent unconscious semantic processing. Advances in Cognitive Psychology, 5,56 .

Mason, M. F., \& Bar, M. (2012). The effect of mental progression on mood. Journal of Experimental Psychology: General, 141(2), 217.

Mednick, M. T., Mednick, S. A., \& Jung, C. C. (1964). Continual association as a function of level of creativity and type of verbal stimulus. The Journal of Abnormal and Social Psychology, 69(5), 511.

Moores, E., Laiti, L., \& Chelazzi, L. (2003). Associative knowledge controls deployment of visual selective attention. Nature Neuroscience, 6(2), 182.

Navon, D. (1977). Forest before trees: The precedence of global features in visual perception. Cognitive Psychology, 9(3), 353-383.

Neely, J. H. (1977). Semantic priming and retrieval from lexical memory: Roles of inhibitionless spreading activation and limited-capacity attention. Journal of Experimental Psychology: General, 106(3), 226.

Nelson, D. L., Bajo, M. T., \& Casanueva, D. (1985). Prior knowledge and memory: The influence of natural category size as a function of intention and distraction. Journal of Experimental Psychology: Learning, Memory, and Cognition, 11(1), 94.

Posner, M. I., \& Snyder, C. R. R. (1975). Attention and cognitive control. In R. L. Solso (Ed.), Information Processing and Cognition: The Loyola Symposium. Lawrence 
Our inherent need for associative coherence

Erlbaum.

Raaijmakers, J. G., \& Shiffrin, R. M. (1981). Search of associative memory. Psychological Review, 88(2), 93.

Rowe, P. G. (1991). Design thinking. MIT press.

Rubinsten, O., Anaki, D., Henik, A., Drori, S., \& Faran, Y. (2005). Free association norms in the Hebrew language. Word Norms in Hebrew, 17-34.

Simonson, E., \& Brozek, J. (1952). Flicker fusion frequency: background and applications. Physiological Reviews, 32(3), 349-378.

Sperling, G. (1960). The information available in brief visual presentations. Psychological Monographs: General and Applied, 74(11), 1.

Sternberg, R. J. (1999). Handbook of creativity. Cambridge University Press.

Summerfield, J. J., Lepsien, J., Gitelman, D. R., Mesulam, M. M., \& Nobre, A. C. (2006). Orienting attention based on long-term memory experience. Neuron, 49(6), 905-916.

Trapp, S., Shenhav, A., Bitzer, S., \& Bar, M. (2015). Human preferences are biased towards associative information. Cognition and Emotion, 29(6), 1054-1068.

Vul, E., Bergsma, J., \& MacLeod, D. I. A. (2010). Functional adaptive sequential testing. Seeing and Perceiving, 23(5), 483-515.

Watson, A. B., \& Pelli, D. G. (1983). QUEST: A Bayesian adaptive psychometric method. Perception \& Psychophysics, 33(2), 113-120. 